AIAA-93-1628

AIAA/ASME/ASCE/AHS/ASC 34th Structures, Structural Dynamics and Materials Conference

April 20-22, 1993, La Jolla, CA

\title{
AEROELASTIC DYNAMICS OF MISTUNED BLADE ASSEMBLIES WITH CLOSELY SPACED BLADE MODES *
}

\author{
Christophe Plerre $\dagger$ \\ Department of Mechanical Engineering and Applied Mechanics \\ The University of Michigan \\ Ann Arbor, Michigan 48109-2125 \\ Durbha V. Murthy $\ddagger$ \\ NASA Lewis Research Center \\ Cleveland, Onio \\ and The University of Toledo \\ Toledo, Ohio
}

\section{ABSTRACT}

The aeroelastic characteristics of tuned and randomly mistuned blade assemblies which possess two blade-alone natural modes with close frequencies are studied. Modal interactlons among the two blade modes are shown to become extremely signiffcant for small frequency separation. The two distinct loci of the aeroelastic eigenvalues, which characterize an assembly with well separated modes, fully merge tnto a single root locus as the blade-mode frequency separation vanishes. Also, while in the case of well separated blade modes the introduction of random mistuning into one blade mode affects only the assembly modes which are predominantly of that blade-mode type, mistuning results in the localization of all the assembly modes when the blade-alone natural frequencies are close. Results indicate that in the case of closely-spaced blade modes a single-degree of freedom blade model yields qualitatively erroneous results and that an $N$-blade assembly with two close blade modes behaves like an equivalent $2 \mathrm{~N}$ blade assembly with a single blade mode.

* This work was supported by NASA Lewis Grant NAG3. 1163 with Dr. G. Stefko as the technical monitor.

† Associate Professor, Member AIAA, Member ASME

† NASA Research Associate, Senior Member AIAA, Member ASME

Copyright (C) 1993 by Christophe Pierre. Published by the American Institute of Aeronautics and Astronautics, Inc. with permission.

\section{INTRODUCTION}

Perfect periodicity, or cyclic symmetry, is a convenient and frequent assumption when analyzing the dynamics of bladed disk assemblies. A primary reason for taking advantage of cyclic symmetry is that the blade excitation and response can always be expressed in terms of constant interblade phase angle modes which uncouple the equations of motion, thereby reducing the size of the problem to that of one blade. This simplification yieids a drastic reduction in computational cost. Such ideal regularity, however, holds true only if all the blades are identical and uniformly spaced and if the disk is symmetric and homogeneous. Perfect periodicity, though, is always disrupted by small differences in the blade structural properties, which result from manufacturing and material tolerances and in-service degradation. Cyclic symmetry of the unsteady aerodynamic loading may also be destroyed by a slightly unequal spacing of the blades or by small variations in the mode shapes of the blades. This phenomenon, commonly known as mistuning, tremendously increases the size, cost, and complexity of the analysis of blade assemblies such as engines and fans.

Numerous research studies have been conducted in an attempt to understand the effects of mistuning on the dynamics of blade assemblies (for example, we refer to the survey paper by Srinivasan [1] and the references contained therein). These studies have shown that while mistuning has a beneffcial, stabilizing effect in a flutter situaton $[2,3]$, it typically has a quite undesirable effect on the 
assembly forced response through a possibly very large increase in the amplitude experienced by some blades [4]. It has also been found that blade mistuning results in the appearance of new peaks in the frequency response [5].

Several recent studies sought to identify the key system parameters which govern the sensitivity of the assembly dynamics to random mistuning (for example, see the works of Valero and Bendiksen [6], Wei and Pierre [7], and Pierre and Murthy [8]). These studies showed that the sensitivity of the aeroelastic dynamics of a blade assembly to mistuning can vary by several orders of magnitude depending on the strength of the interblade coupling (whether structural or aerodynamic or both), the excitation frequency, and the number of blades. Perhaps the most important finding was that weak aerodynamic or structural interblade coupling results in close aeroelastic eigenvalues, high sensitivity to mistuning, and qualitative alterations of the blade assembly dynamics. Specifically, in the high sensitivity case both the free and forced vibration responses were found to become localized to a small geometric region of the assembly (that is, to a few blades), a phenomenon which generally leads to a severe amplitude increase for some of the blades. Associated with the phenomenon of mode localization is that of root locus scattering, in which the locus of the aeroelastic eigenvalues (frequency versus damping) loses the regular pattern that characterizes a tuned assembly to become apparently randomly scattered for small random mistuning.

The above general trends regarding mistuning effects and the assoclated physical insights into the dynamics of mistuned assemblies were generated through the application and development of perturbation methods to the aeroelastic eigenvalue problem $[7,8]$. The perturbation approach capitalized on the existence of two small param. eters for highly sensitive blade assemblies, namely the random mistuning and the interblade coupling. It was shown that it is the combined effects of these two small parameters which is the cause of high sensitivity to mistuning and mode localization.

In the proposed paper we examine the effects of a third small parameter on the sensitivity of blade assemblies to mistuning, namely the frequency separation between the natural modes of a blade. Typically, the modal interactions among the various natural modes of a blade are weak unless some modes feature close frequencies. When all the natural frequencies are well-separated, each blade can be effectively approximated as a single-degree of freedom system for each of the blade modes of interestan approach widely adopted. However, situations may arise in which two blade modes feature close frequencies, for example a bending mode and a torsion mode of a blade, or two of the modes of a plate-like blade with low aspect ratio (recall that depending on geometry, plates may feature a number of close or even repeated natural frequencies). In such cases one can anticipate significant interactions among the close blade modes, due to aerodynamic coupling and mistuning effects. The aim of the proposed parer is to study the consequence of these modal interactions on the sensitivity of the assembly to mistuning and the localization of the aeroelastic modes.

To date, most research studies of mistuning have considered single-degree of freedom oscillator modeis of the blades, thereby implicitly assuming large frequency separation of the blade modes. In a few mistuning studies, coupling among blade natural modes was accounted for, but also for well separated natural frequencies $[9,10]$. Kaza and Kielb [2] examined the effect of varying the ratio of the bending to torsion natural frequencies of the blades on the flutter speed, but to date no systematic examination of mistuned assemblies with close blade modes has been performed.

In this paper we study the aeroelastic characteristics of tuned and randomly mistuned blade assemblies which possess two blade-alone natural modes with close frequencies. Only (weak) aerodynamic coupling among the blades is considered. Both the scattering of the locus of the complex eigenvalues and the localization of the associated mode shapes are examined as descriptors of the sensitivity to mistuning. The existence of three small parameters for this problem - the interblade coupling, blade mode separation, and random blade mistuning-allows us to formulate physical interpretations of and identify similarities between the effects of blade mode separation and blade mode mistuning. It is shown in the paper that the modal interactions between the two blade modes become extremely significant for small frequency separation. Further, the effects of small frequency separation are shown to be easily understood by invoking analogies to equivalent assemblies of single-degree of freedom blades. Specifically, it is argued that the blade mode frequency separa. tion can be regarded as a form of frequency mistuning, and that an $N$-blade rotor with two close blade modes can be viewed either as a $2 \mathrm{~N}$-bladed rotor with alternate "mistuning" (which corresponds to the small blade mode separation) or as two coupled $\mathrm{N}$-blade rotors-one corresponding to each blade mode.

The paper is organized as follows. The equations of aeroelastic motton are formulated in Section 2 for tuned and randomly mistuned rotors with two degrees of freedom per blade. The aeroelastic eigensolution of the tuned rotor is discussed in Section 3 as a function of the blade mode frequency separation, and the interpretation of mode separation as a mistuning parameter is formulated. Section 4 describes the results obtained for the randomly mistuned rotor. Root locus scattering and mode localization phenomena are examined in terms of blade mode separation. The paper's conclusions are given in Section 5.

\section{EQUATIONS OF AEROELASTIC MOTION FOR MULTT- DEGREE OF FREEDOM PER BLADE FORMULATON}

The structure we examine consists of $N$ blades equally spaced on a rigid disk. Hence there is no structural coupling among the blades. Each blade is modeled as a "typical section," with one bending and one torsion degree of 
freedom. The model of the blade and that of the blade assembly are taken from the work of Kielb and Kaza [2].

The center of gravity of the typical section is taken to coincide with its elastic axis, which results in completely uncoupled torsion and bending natural modes for a biade in a vacuum. This allows us to vary the ratio of the bending natural frequency to the torsional natural frequency of a blade, simply by changing the stiffness of the torsional or the translational spring of the typical section. Hence the two blade modes are completely uncoupled structurally, and the only bending-torsion coupling originates from unsteady, motion-dependent aerodynamic effects. This setup allows us to vary the separation of the blade mode frequencies in a controlled manner.

The unsteady, motion-dependent aerodynamic forces are calculated by applying two-dimensional, linear, unsteady, cascade aerodynamic theories for the subsonic regime considered here [11]. Note that the effects of airfoil thickness, camber and steady incidence are neglected and that the flow is assumed to be isentropic and irrotational. This calculation results in a complex matrix of generalized aerodynamic coefficients. Note that motion-independent aerodynamic forces and structural energy dissipation are not included in our model.

The above model formulation yields a system of $2 \mathrm{~N}$ linear, homogeneous, ordinary differential equations in the $N$ blade torsion amplitudes and the $N$ bending amplitudes. We seek motions such that all the blade coordinates osclllate with the same frequency and/or decay or grow at the same rate. This yields the aeroelastic eigemvalue problem.

$$
\left\{-\lambda\left(\mathbf{M}+\mathbf{A}\left(\omega_{a}\right)\right)+\mathbf{K}\right\} \mathbf{u}=\mathbf{0}
$$

where

- $\mathbf{u}=\left[h_{1} / b, \alpha_{1}, h_{2} / b, \alpha_{2}, \ldots, h_{N} / b, \alpha_{N}\right]^{T}$ is the $2 N$ dimensional complex eigenvector of the blade amplitudes, where $T$ denotes a transpose, $h_{i}$ is the bending amplitude of $i$ th blade normalized by the blade semichord, $b$, and $\alpha_{i}$ is the $i$ th blade torsion amplitude.

- $M$ and $K$ are $2 N$ by $2 N$ mass and stuffness matrices, respectlvely.

- A is the $2 N$ by $2 N$ complex aerodynamic matrix, calculated at the assumed frequency $\omega_{a}$.

- $\lambda$ is the complex eigenvalue.

The matrices $\mathrm{K}, \mathrm{M}$, and A consist of $N^{2}$ blocks, each of size two by two. Since there is no structural coupling between blades, $K$ and $M$ are block-diagonal matrices, where the blocks on the diagonal are the stiffness and mass matrices of the individual blades, respectively. Furthermore, since in this work the center of gravity of the typical section is taken to coincide with its elastlc axis, there is no structural coupling between the bending and torsion motons for an individual blade (in a vacuum), which means that the blocks which make up $K$ and $M$ are themselves dfagonal. Hence the assembly stiffness matrix can be written as:

$$
\mathbf{K}=\left[\begin{array}{llll}
\mathbf{K}_{1} & & & \\
& \ddots & 0 & \\
& \mathbf{0} & \ddots & \\
& & & \mathbf{K}_{N}
\end{array}\right]
$$

where

$$
\mathbf{K}_{i}=\left[\begin{array}{cc}
\omega_{b i}^{2} & 0 \\
0 & \omega_{t i}^{2}
\end{array}\right] \quad i=1, \ldots, N
$$

where $\omega_{b i}$ and $\omega_{t i}$ are the bending and torsion natural frequencies of the $i$ th blade, respectively. (Equation (3) holds only if both modes of all individual blades have been normalized, in which case the mass matrix $M$ simply equals the $2 N$ by $2 N$ identity matrix) Here frequency mistuning has been assumed. For a tuned system all bending blade natural frequencies are equal, $\omega_{b i}=\omega_{b}$, and all torsion frequencies are equal, $\omega_{t i}=\omega_{t}$. For a mistuned assembly the blade bending frequencies, or the torsion frequencies, or both are random. Also note that with the assumption of frequency mistuning the mass matrix consists of identical blocks on the diagonal (these blocks being themselves diagonal).

While the assembly mass and stiffness matrices are diagonal, the aerodynamic matrix $A$ is fully populated. The off-diagonal blocks of A provide aerodynamic coupling between the blades, while the off-diagonal elements of each block (including of the blocks on the diagonal) account for the aerodynamic coupling between the bending and torsion blade modes.

When the system is assumed to be tuned, i.e., the blades are all identical, all matrices are block-circulant, where the dimension of each block equals the number of blade modes, that is, two. In the tuned case the blade motion in each normal mode of free vibration is simple harmonic in space as well as in time. In a normal mode motton, all blades vibrate with equal amplitude and with a constant phase angle between adjacent blades. Due to cyclic symmetry, this interblade phase angle is restricted to take $N$ discrete values, $\sigma_{n}=2 \pi(n-1) / N, n=1, \ldots, N$. Hence for each admissible value of the interblade phase angle there are two free vibration modes of the tuned assembly-for example, but not necessarlly so, one mode featuring predominantly bending blade motion and the other mostly torsion blade motion.

For an assembly with blade frequency mistuning, however, the stiffness matrix is still diagonal but no longer block circulant, signifying the departure of the assembly modes from constant interblade phase angle modes (note that the mass and aerodynamic matrices remain blockcirculant).

The solution of the aeroelastic eigenvalue problem, Eq. (1), dictates the nature and stability of the assembly's motion in an aeroelastic mode. For an eigensolution $(\lambda, u)$, the blade assembly's motion is given by $u \exp (i \omega t)$, with $\lambda=\omega^{2}$ and $i^{2}=-1$, where $\omega$ is the complex frequency defined by $i \omega=\mu+i \nu$. In this paper we shall refer to $\mu$. 
the rate of exponential growth of the blade motion amplitude, as the "real part of the eigenvalue." Note that flutter instability occurs in a mode when $\mu>0$. The frequency of oscillations, $\nu$, is referred to in the paper as the "imaginary part of the eigenvalue." The complex eigenvectors provide the $2 \mathrm{~N}$ amplitudes and phases of the aeroelastic mode shapes.

Detailed expressions for the matrices in Eq. (1) can be found in reference [2]. Also, the parameters used for generating the results of this paper are given in Table 1.

In the remainder of the paper we examine how the aeroelastic modes of the assembly, obtained by solving Eq. (1), are affected by the closeness of the bending and torsion natural frequencies of a blade and by the introduction of small random mistuning in the bending or torsion natural frequencies of the various blades.

\section{TUNED SYSTEM RESPONSE FOR CLOSELY-SPACED BLADE MODES}

Here we examine the effect of blade mode frequency separation on the aeroelastic eigenvalues and mode shapes of the tuned assembly.

Figure 1 depicts the root locus of the aeroelastic eigenvalues (natural frequency versus damping factor) of a tuned assembly with 56 blades, for various values of the ratio of the blade-alone bending frequency to the torsion frequency. Since in this model there are two natural modes per blade, the system possesses 112 eigenvalues. The corresponding mode shapes all feature a constant interblade phase angle, such that there are two modes for each admissible value of the interblade phase angle $\left(\sigma_{n}=2 \pi(n-1) / N, n=1, \ldots, N\right)$. The two mode shapes for each $n$ feature each torsion and bending modal amplitudes which are constant throughout the rotor, but in general the ratios of the bending amplitude to the torsion amplitude differ for the two modes.

Observe in Fig. Ia that when the two blade mode frequencies are not too close (here the bending to torsion frequency ratio equals 0.9 ) the aeroelastic eigenvalues form two distinct loci of 56 eigenvalues each. There is one mode with a given interblade phase angle in each locus. The ratios of the torsion amplitude to the bending amplitude, indicated for some modes in Fig. 1a, reveal that the upper locus features modes in which the assembly vibrates primarily in the blade torsion mode, while the lower locus corresponds mostly to a bending motion of the blades. Nevertheless, note that there is a small contributton of the bending blade mode to the assembly modes of the upper (torsion) locus and vice versa. This phenomenon is due to the coupling of the two blade modes through the unsteady aerodynamic forces. This modal coupling, though clearly not sufficient to merge the two distinct loci in Fig. 1a, affects the blade modal amplitudes significantly. This is because the blade torsion and bending frequencies are not strongly separated in this case. The modal coupling effect is most pronounced for the modes of one locus which are closer to the other locus (for example, the low frequency modes of the torsion locus), as these are observed to feature a large contribution from the other blade mode. Also note in Fig. Ia that the spread in the natural frequencies for each locus represents the amount of aerodynamic coupling among blades for that particular mode group. This implies that for our system the coupling among blades which vibrate in their torsion mode is stronger than that among blades undergoing a bending motion, although the aerodynamic coupling is obviously weak for both blade modes. Finally, note that the root locus displayed in Fig. 1a is quite similar that of an assembly with alternate blade mistuning [2]. Hence we can view the $N$-blade assembly with two blade modes as an equivalent assembly of $2 \mathrm{~N}$ single-mode blades, with every other blade featuring a torsion mode frequency and the others a bending mode frequency-thus corresponding to an alternate bending/torsion "mistuning." Alternatively, we can regard the root loci depicted in Fig. 1a as those of two weakly coupled, dissimilar assemblies of $N$ blades each-one "bending mode assembly" and one "torsion mode assembly," such that the coupling between the two subsystems is not sufficiently large to overcome the frequency separation between the bending- and the torsion-dominated motions which characterize each "assembly." The structure of the equations of motion derived in Section 2 supports these conjectures.

Figures 1b-d show that as the blade-alone bending to torsion frequency ratio increases, the two distinct loci come closer together and gradually merge. Indeed, for bending to torsion frequency ratios between 0.94 and 0.97 , the aeroelastic eigenvalues constitute a single locus of 112 eigenvalues, which would be characteristic of an equivalent assembly of 112 blades with a single mode per blade. One difference between the locus of the assembly with 56 blades and two blade modes and that of an assembly with 112 single-mode blades, however, is that in the former there are only 56 distinct interblade phase angles, with two modes for each phase angle value, while the latter features 112 distinct interblade phase angles. The merging of the two root loci observed in Figs. Ib-d occurs because the aerodynamic coupling between the bending and torsion modes become predominant as the frequency separation between these two blade modes decreases. Indeed, as frequency separation decreases, the assembly mode shapes become less dominated by either the bending or the torsion component to feature a significant contribution from both blade modes. In particular, the two assembly modes for each interblade phase angle become more alike as frequency separation decreases and thus as coupling between blade modes through the aerodynamic terms increases. This is confirmed by the torsion to bending amplitude ratos listed for some modes in Figs. 1b-d, which are generally closer to the value of one than in Fig. 1a. (Here it is worth pointing out that all pairs of interblade phase angle modes do not feature strongest coupling, that is, closest bending to torsion amplitude ratios within a mode pair, for the same value of the bending to torsion frequency 
ratio; hence the still relatively wide range of amplitude ratios observed in Figs, $1 \mathrm{~b}-\mathrm{d}$.) As the bending to torsion frequency keeps increasing, the root locus is observed to separate gradually into two distinct loci in Figs. le and If, signifying the decrease of blade mode coupling through the aerodynamic terms as the bending and torsion frequencies move apart. Note that for the larger bending to torsion frequency ratios the bending-dominated locus becomes the upper locus. Hence a switching of the two root loci effectively occurs as the frequency ratio increases. Finally, it is worth mentioning that the merging of the two loci is not exactly centered at a frequency ratio of one, but rather near 0.95 . This is most probably due to the fact that aerodynamic terms provide not oniy coupling between the blade modes but also additional stiffness to the individual blade modes, and that the bending and torsion modes may not be identically affected by these aerodynamic terms.

Returning to the analogy to a $2 \mathrm{~N}$-blade assembly with altermate bending/torsion mistuning invoked above, the merging of the loci observed in Figs. $1 \mathrm{~b}-\mathrm{d}$ can be explained by noting that as the blade mode frequency separation decreases, the alternate "mistuning" strength decreases and thus the coupling between blade modes becomes dominant. As the blade mode frequency separation (which ought to be defined by appropriately accounting for the added aerodynamic stlffness) vanishes, the "mistuning" vanishes as well, yielding a 112-blade "tuned" assembly which features a single root locus, as shown in Figs. 1b-d. As the blade mode separation increases in Figs. Ie and 1f, the alternate mistuning grows again and the aerodynamic coupling between the blade modes is not sufficient to keep the loci merged, and separation is observed.

The merging of the root locl can also be interpreted with the analogy to two assemblles with $N$ blades each, one corresponding to the bending blade mode and the other to the torsion blade mode. As the blade mode frequency separation decreases, the two $N$-blade assembltes become increasingly similar and, relatively, the inter-assembly aerodynamic coupling increases. This means that the blade bending and torsion modes become increasingly coupled, causing all assembly modes to feature significant bending and torsion components, much in the same way as the modes of a system of two coupled, slightly dissimilar oscillators feature oscillations of both oscillators as coupling increases. As blade mode frequency separation is increased in Figs. Ie and 1f, the "mistuning" between the two $N$-blade sub-assemblies grows and the assembly modes resume being either bending- or torsion-dominated, similarly to the localized modes of a weakly coupled, mistuned two-oscillator system [12].

In order to further illustrate the effect of coupling among blade modes as frequency separation varies, FIg. 2 displays the bending to torsion amplitude ratio versus the bending to torsion frequency ratio for the pair of interblade phase angle modes $n=18$. Observe how the torsion- and bending-dominated assembly modes, which are characteristic of the larger frequency separation, be- come a nearly equal mix of bending and torsion compo. nents as the two blade-alone frequencies approach each other, with the strongest blade mode coupling occurring for a frequency ratio equal to about 0.98 . Note that for this mode pair the strongest coupling occurs slightly after the two loci have separated. This point of strongest coupling was observed to depend slightly on the interblade angle considered-again this is because of the stiffness added by the aerodynamic terms, which depends on the interblade phase angle. It is also noteworthy that the bending to torsion amplitude ratios are not equal to one for strong blade mode coupling. This is not surprising since the bending and torsion modal amplitudes represent physically different motions, hence equal contributions to the motion from the bending and torsion components do not necessarily imply equal modal amplitudes.

The effect of coupling between bending and torsion blade modes is further illustrated in Fig. 3, which depicts the loci of the aeroelastic frequencies for the $n=18$ pair of modes in terms of the blade mode frequency separation. The phenomenon of curve veering is observed, such that the two aeroelastic frequency loci approach each other as blade mode separation is reduced, and then veer away from each other as the bending to torsion frequency ratio is increased (stronger or weaker curve veerings would be observed for the other pairs of constant interblade phase angle modes). The curve veering in Fig. 3 is similar to that obtained for a system of two coupled oscillators [12], indicating the validity of the analogy to the two $N$-blade subassemblies described above. The interaction between the bending and torsion motions is strongest in the veering region, thereby confirming the merging of root loci in Fig. 1.

\section{RANDOM MISTUNING AND MULTHDEGREE OF FREEDOM PER BLADE RESPONSE}

The rotor assembly can be mistuned either by altering the blade-alone torston natural frequenctes or the bending frequencles or both. The blade mistuning considered here is random with a uniform probability distribution, and it is characterized by its small standard deviation. As an illustration of the effects of random blade frequency mistuning on the aeroelastic modes of the assembly, Fig. 4 displays the root locus of the eigenvalues for a bending to torsion frequency ratio of 0.99 . Both a tuned assembly and one with mistuning of standard deviation $0.3 \%$ in the blade-alone bending natural frequencies are considered. In the tuned state the eigenvalues form two close but distnct loci, and it is apparent that mistuning affects only the upper locus in a noticeable way, which corresponds mostly to a bending vibration of the blades. Specifically, observe the scattering of the bending-dominated locus as the strength of bending frequency mistuning increases. The phenomenon of root locus scattering was shown by Pierre and Murthy [8] to be characteristic of highly sensitive mistuned blade assemblies and to signify the occurrence of severe mode localization. Hence we expect the mode shapes associated with the eigenvalues of the upper locus to be strongly localized to a few of the 56 blades 
due to the small mistuning. On the other hand, notice that the torsion-dominated (lower) locus remains quite regular when mistuning is introduced. We reckon that the corresponding mode shapes conserve a constant interblade phase angle and extend throughout the assembly.

In the case of Fig. 4, the introduction of mistuning in the blade bending frequencies only influences the bending-dominated locus because the aerodynamic coupling between the two blade modes is fairly weak. Thus the torsion-dominated modes remain relatively unaffected by the bending frequency mistuning. Similariy, one expects that mistuning the torsion blade frequencies would only affect the lower root locus to any significant degree. The conclusion is that in the case of distinct root loci for the two blade modes, the effects of mistuning on the assembly dynamics are similar to those on two uncoupled assemblies, one for each blade mode. Hence for weak coupling between blade modes, two separate analyses of assemblies with a single degree of freedom per blade can be performed-an expected result. This is the case because coupling between the two $N$-blade sub-assemblies is too weak for mistuning in one assembly to affect the other, or equivalently, because the blade-mode separation is large compared to the bending mode mistuning.

To confirm these findings, Fig. 5 depicts the frequency loci and selected mode shapes for a randomly mistuned assembly with a bending to torsion frequency ratio equal to 0.9 (case of Fig. 1a) and with $1 \%$ bending frequency mistuning. Observe the scatter of the bending-dominated root locus, while the torsion-dominated locus remains regular and little affected by mistuning. Furthermore, note that the mode shapes that are bending-motion dominated fundamentally change character from the tuned modes to become strongly localized about a few blades. Mode shape localization and root locus scattering were shown in reference [8] to be two manifestations of the same phenomenon; this is confirmed in Fig. 5 for the bendingdominated aeroelastic modes. Conversely, the torsiondominated mode shapes in Fig. 5 are little affected by mistuning and feature a nearly uniform amplitude throughout the assembly. This is consistent with the regular root locus of the torsion-dominated modes. Back to the bendingdominated modes, notice that the localization of the mode shapes is less pronounced for the natural frequencies which are closer to the torsion-mode locus. This is because these mode shapes feature a greater, though still small, torsion participation, thereby making them slightly less sensitive to bending-mode mistuning.

Figure 6 displays the effects of torston frequency mistuning on the root locus of the assembly, for a bending to frequency ratio of 0.965 and various mistuning strengths. In this case the tuned assembly features a merged locus, corresponding to blade modes which are strongly coupled through the aerodynamics. Contrary to the behavior shown in Fig. 4, the root locus in Fig. 6 gradually loses its regular structure to become randomly scattered as mistuning strength increases. Although the mistuning is re- stricted to the torsion natural frequencies of the blades, the entre locus, hence all of the 112 aeroelastic modes, seem to be similarly affected by mistuning. This can be explained by noting that the strong blade mode coupling causes all assembly modes to have a significant torsion component and hence to be strongly affected by torsion mistuning. The corresponding aeroelastic mode shapes are depicted in Fig. 7 for a mistuning of strength $2 \%$. The figure reveals that all the modes seem to be strongly affected by torsion-mode mistuning and high levels of localization are observed. Therefore in this case the assembly appears to behave like an equivalent randomly mistuned assembly with $2 \mathrm{~N}$ blades. It is, however, interesting to note that the localization is not uniform for all mode shapes: in Fig. 7 the mode shape which belongs to the more regular portion of the locus features less severe localization than those which are associated with the "scattered" regions of the root locus. This is again consistent with the findings of Pierre and Murthy [8].

\section{CONCLUSIONS}

The aeroelastic characteristics of tuned and randomly mistuned blade assemblies with two closely-spaced blade modes have been examined. The following conclusions can be drawn:

- Modal interactions among the two blade modes become extremely significant for small frequency separation. The effects of random mistuning are governed by the relative strengths of the interblade coupling, blade mode separation, and blade mistuning.

- Blade-mode frequency separation can be regarded as a form of frequency mistuning, and an $N$-blade ro. tor with two close blade modes can be viewed as a fictitious $2 \mathrm{~N}$-bladed rotor with alternate "mistuning" (which corresponds to the small blade mode separa. tion).

- As blade-mode frequency separation vanishes, the fictttlous $2 \mathrm{~N}$-blade rotor becomes "tuned," and the two root loci of the aeroelastic eigenvalues, which charactertze the assembly with well separated modes, fully merge into a single root locus.

- While in the case of well separated blade modes, the introduction of random mistuning into one blade mode affects only the assembly modes which feature predominantly that blade mode, in the case of close blade-mode frequencies, mistuning results in the localization of all the assembly modes and in the scattering of the entire root locus.

\section{REFERENCES}

1. Srinivasan, A. V., "Vlbrations of Bladed-Disk Assemblies-A Selected Survey," Joumal of Vibration, Acoustics, Stress, and Rellability in Design, 106, 1984, 165168.

2. KJelb, R E and Kaza, K. R. V., "Aeroelastic Characteristics of a Cascade of Mistuned Blades in Subsonic and Supersonic Flows," Jourmal of Vibration, Acoustics, Stress, and Rellability in Design, 105, 1983, 425-433. 
3. Bendiksen, O. O., "Recent Developments in Flutter Suppression Techniques for Turbomachinery Rotors," AIAA Joumal of Propulsion and Power, 4(2), 1988, 164. 171.

4. El-Bayoumy, L E. and Srinivasan, A. V., "Influence of Mistuning on Rotor-Blade Vibrations," ALAA Joumal, 13(4), 1975, 460-464.

5. Ewins, D. J., "Vibration Characterisuics of Bladed Disc Assemblies," Joumal of Mechanical Engineering Science, 15(3), 1973, 165-186.

6. Valero, N. A. and Bendiksen, O. O., "Vibration Characteristics of Mistuned Shrouded Blade Assemblies," Journal of Engineering for Gas Turbines and Power, 108(2), 1986, 293-299.

7. Wei, S. T. and Pierre, C., "Localization Phenomena in Mistuned Assemblies with Cyclic Symmetry Part I: Free Vibrations," Joumal of Vibration, Acoustics, Stress, and Reliability in Design, 110, 1988, 429-438.

8. Pierre, C. and Murthy, D. V., Aeroelastic Modal Characteristics of Mistuned Blade Assemblies: Mode Localization and Loss of Eigenstructure," AIAA/ASME/ASCE/AHS/ASC 32nd Structures, Structural Dynamics and Materials Conference, Baitimore, AIAA Paper 91-1218, 1991.

9. Kaza, K. R. V., Mehmed, O., Moss, L. A. and Williams, M. H., "Analytical and Experimental Investigation of Mistuning on Propfan Flutter," NASA TM-88959, AIAA/ASME/ASCE/AHS 28th Structures, Structural Dynamics and Materials Conference, Monterey, CA, 1987.

10. Kaza, K. R. V., Williams, M. H., Mehmed, O. and Narayanan, G. V., "Aeroelastic Response of Metallic and Composite Propfan Models in Yawed Flow, "NASA TM100964, ALAA/ASME/SAE/ASEE 24th Joint Propulsion Conference, Boston, MA, 1988.

11. Rao, B. M. and Jones, W. P., "Unsteady Airloads on a Cascade of Staggered Blades in Subsonic Flow, ${ }^{n} 46$ th Propulsion Energetics Review Meeting, Monterey, CA, 1975.

12. Pierre, C., "Mode Localization and Eigenvalue Loci Veering Phenomena in Disordered Structures," Journal of Sound and Vibration, Vol. 126, 1988, p. 485-502.

\begin{tabular}{|c|r|}
\hline Random mistuning distribution & Uniform \\
\hline Number of blades & 56 \\
\hline Gap to chord ratio & 0.534 \\
\hline Stagger angle & $54.4^{\circ}$ \\
\hline Mass ratio & 258.5 \\
\hline Inlet Mach number & 0.5 \\
\hline Reduced frequency & 0.469 \\
\hline Radius of gyration & 0.577 \\
\hline Elastic axds location & at mid-Chord \\
\hline Center of gravity location & at mid-chord \\
\hline Structural Damping & 0 \\
\hline
\end{tabular}

Table 1 Model parameters (see reference [2]). 


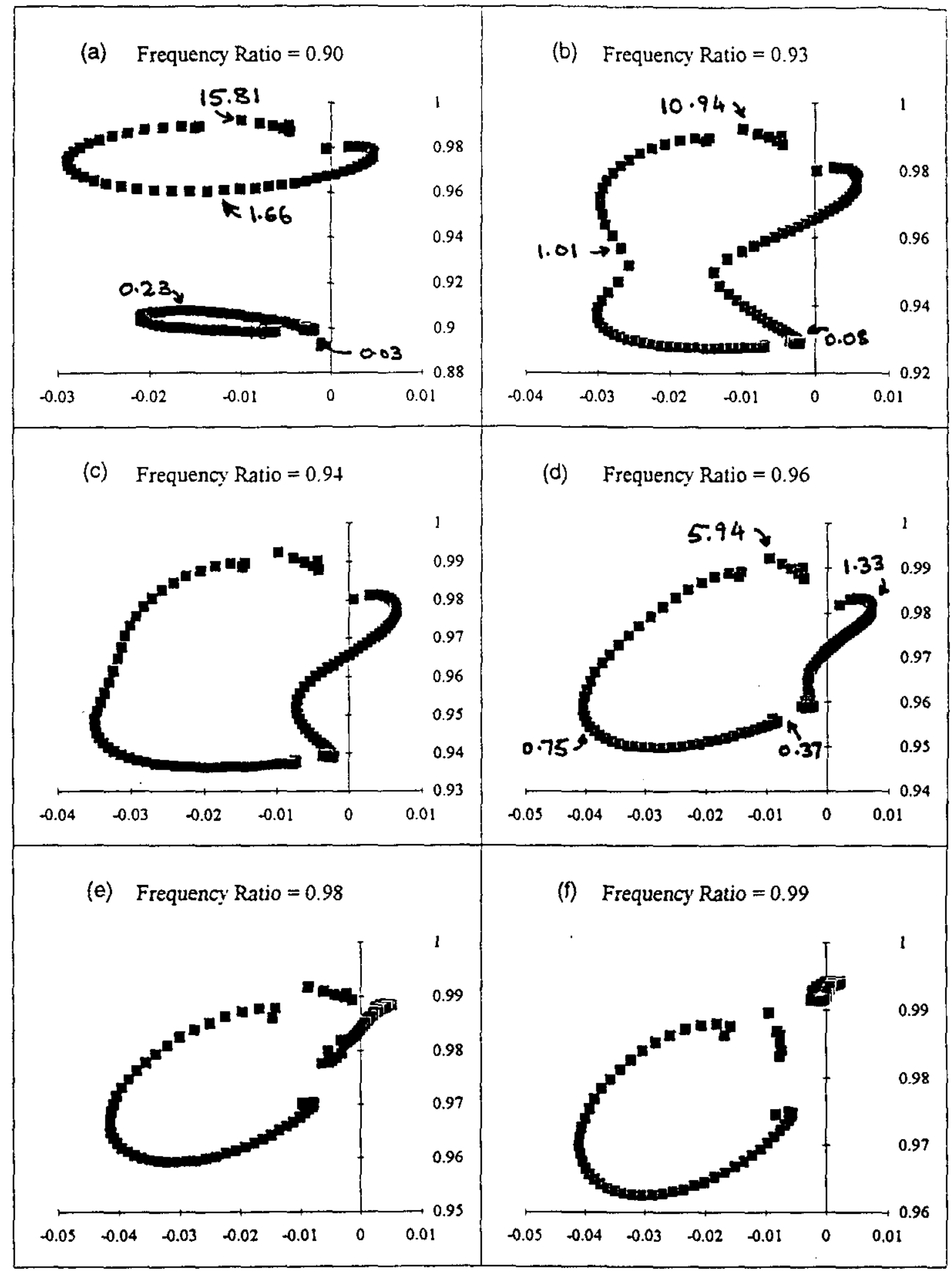

Figure 1. Transformation of root loci as blade-alone natural frequencies approach each other (dimensionless frequency $(v)$ on vertical axis versus dimensionless damping $(\mu)$ on horizontal axis). The ratio of torsion amplitude to bending amplitude is given for selected modes. The frequency ratio is that of the blade-alone bending frequency to the torsion frequency. 
Interblade Phase Angle Index $=18$

Bending/Torsion Amplitude Ratio

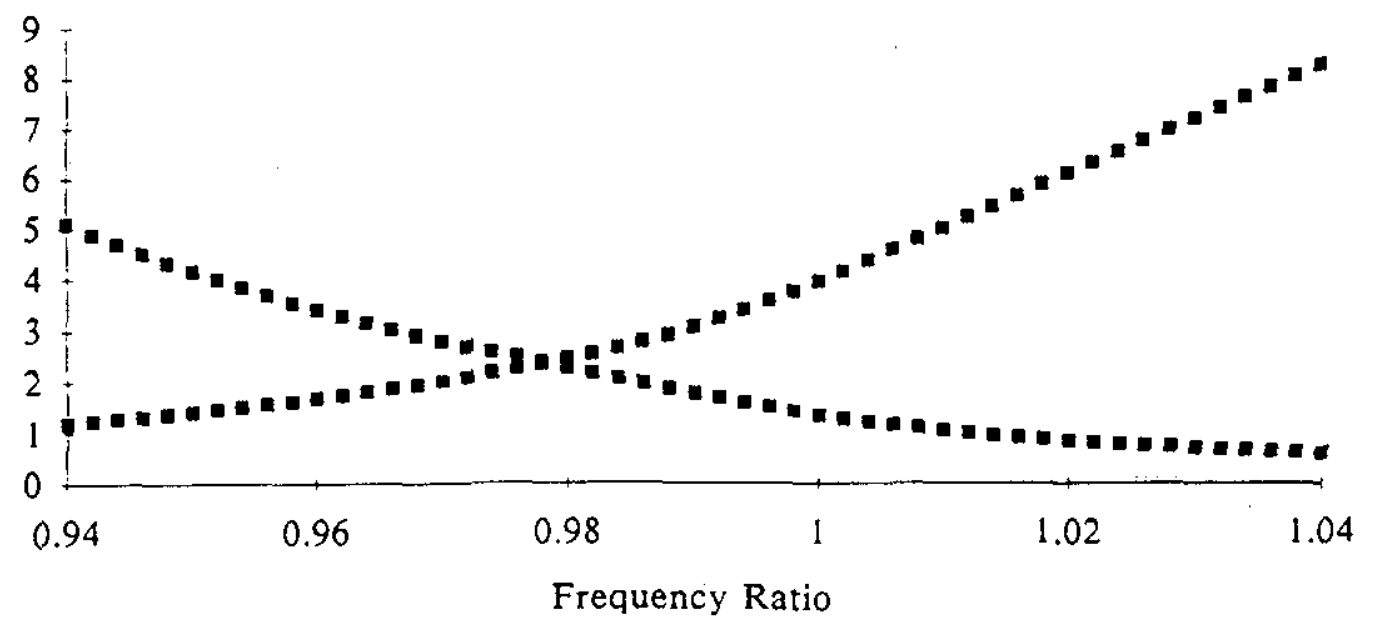

Figure 2. Variation of the bending to torsion amplitude ratlo as a function of the blade-alone bending to torsion frequency ratio, for the $n=18$ pair of constant interblade phase angle modes.

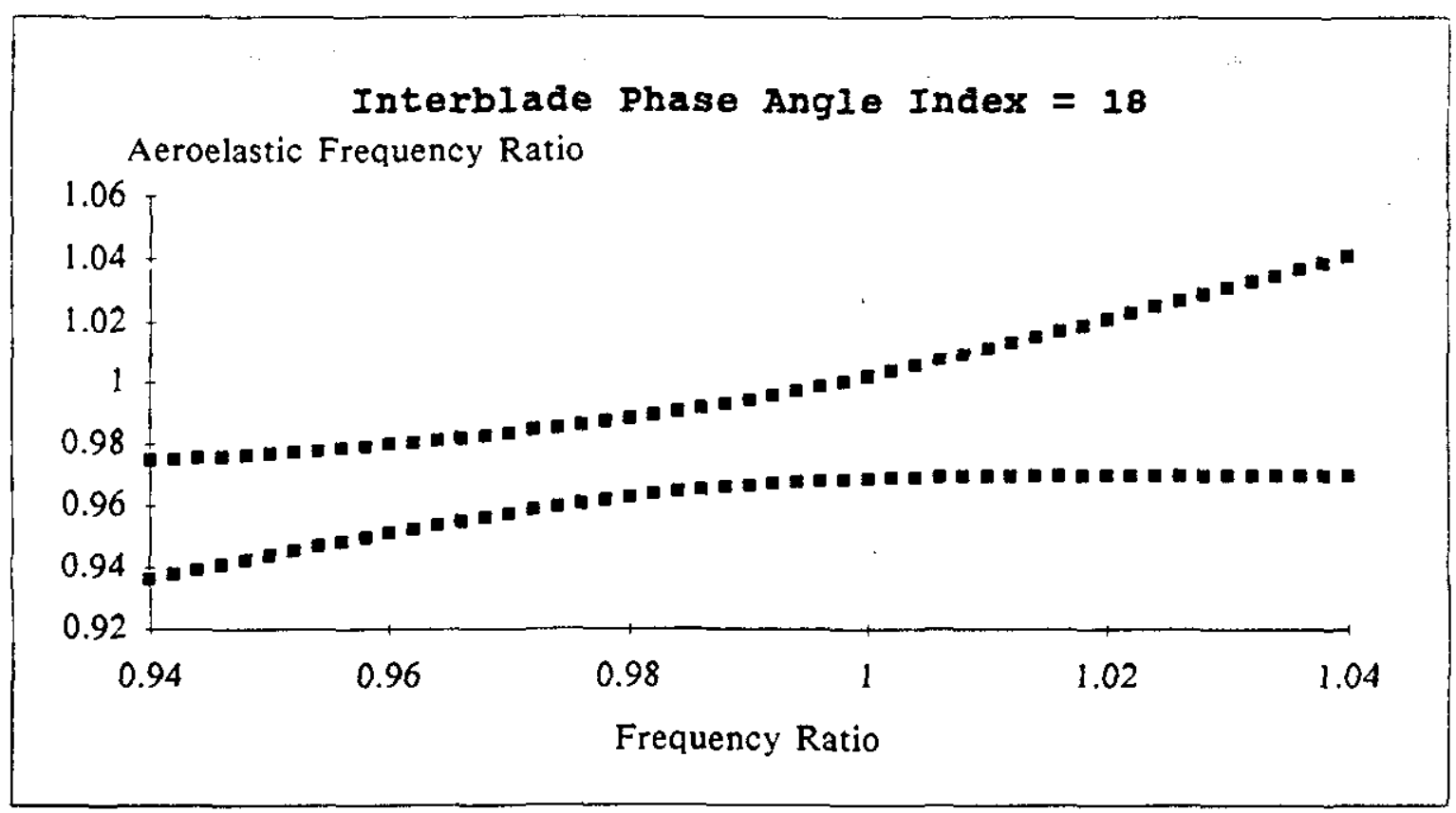

Figure 3. Variation of the assembly aeroelastic natural frequencies $(\nu)$ as a function of the blade-alone bending to torsion frequency ratio, for the $n=18$ pair of constant interblade phase angle modes. 


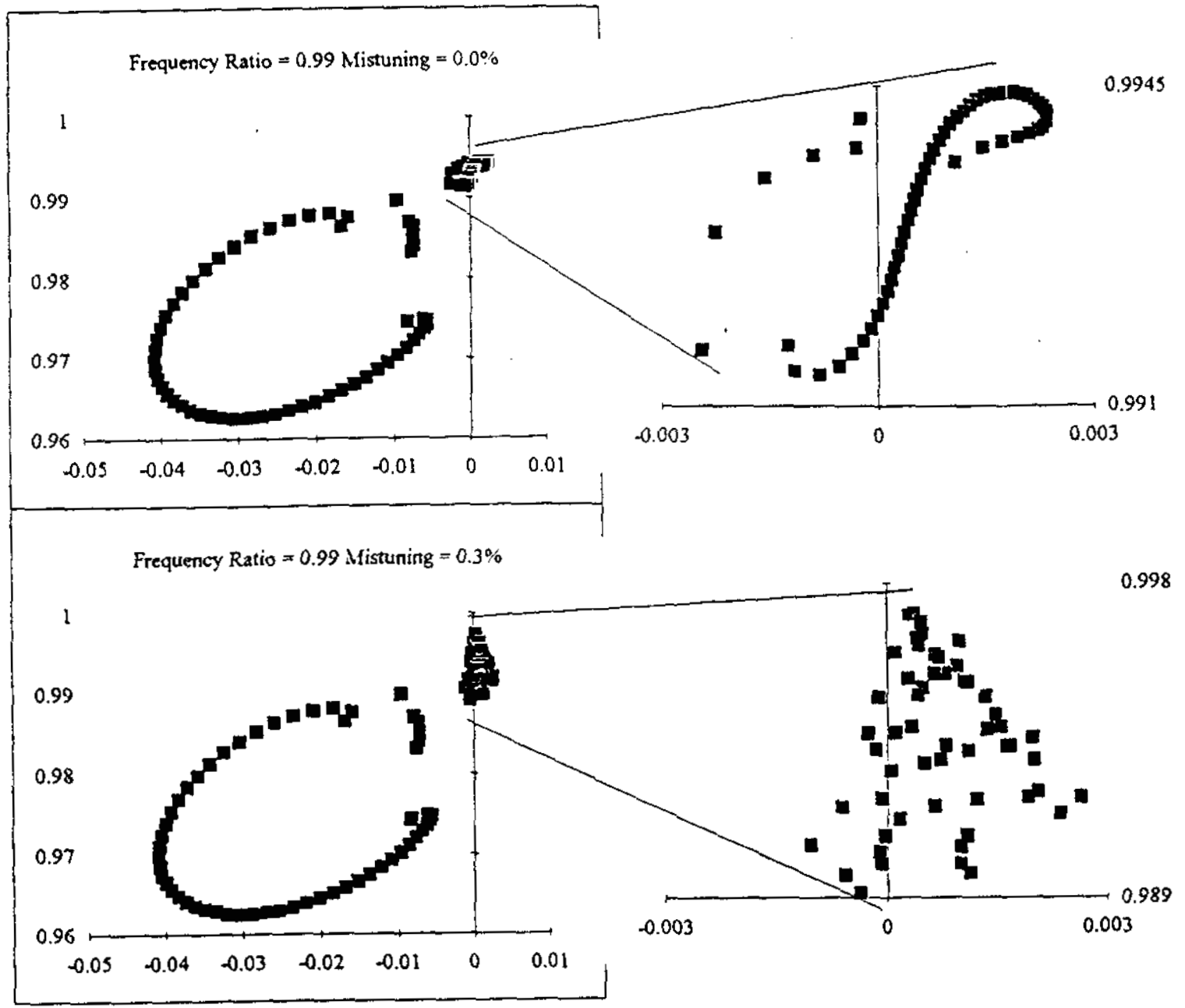

Figure 4. Scattering of the regular structure of the bending root locus by the introduction of random mistuning in the blade-alone bending natural frequencies (axes are as in Fig. 1). The blade-alone bending to torsion frequency ratio is 0.99 and in the mistuned case the mistuning standard deviation ts $0.3 \%$. 


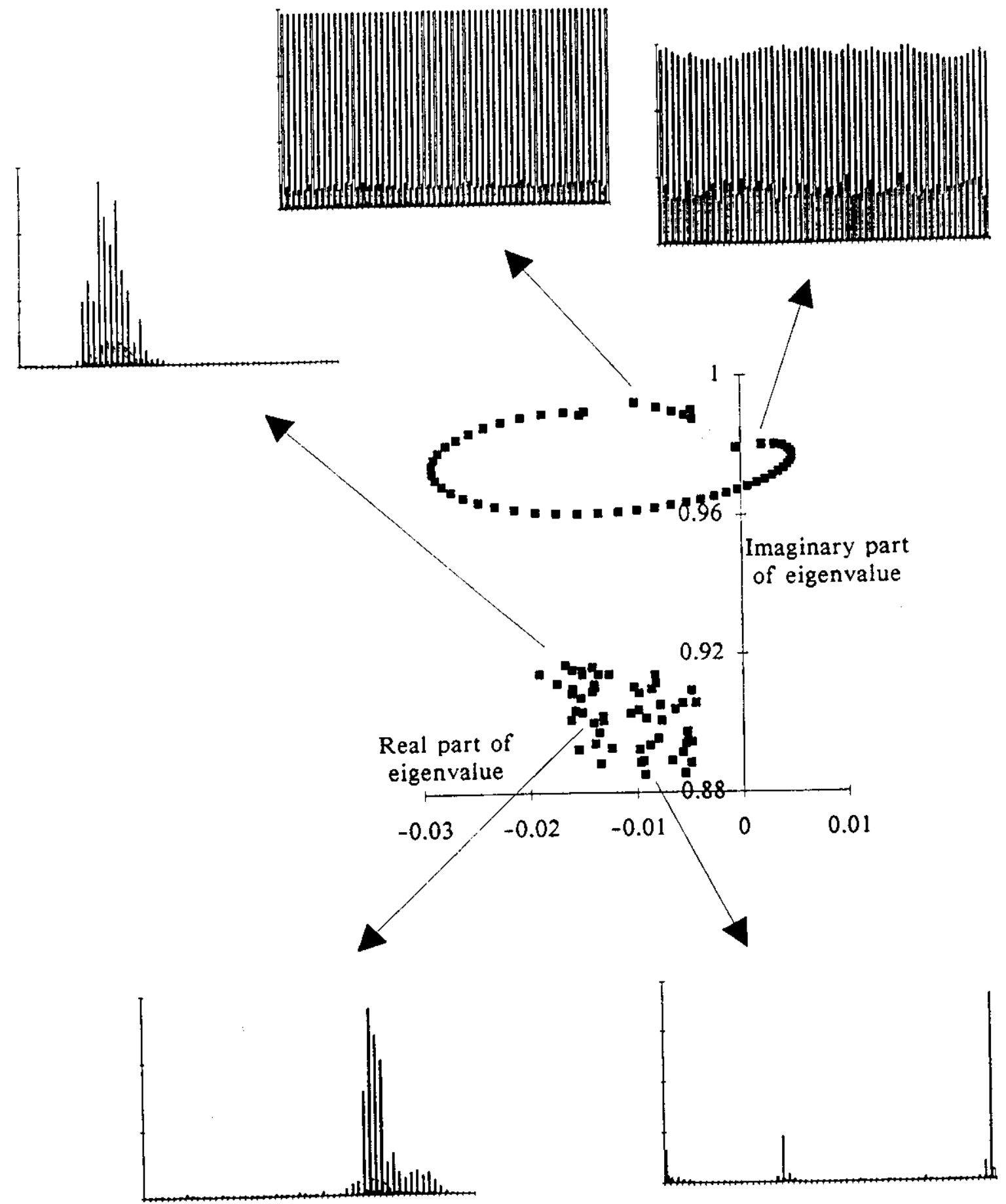

Figure 5. Localization of the mode shapes associated with the scattered bending root locus. The blade-alone bending to torsion frequency ratio is 0.9 and the random mistuning in the blade-alone bending natural frequencies has standard deviation 1\%. For the mode shapes, bending amplitudes (black bars) and torsion (white bars) are plotted versus the blade number. 


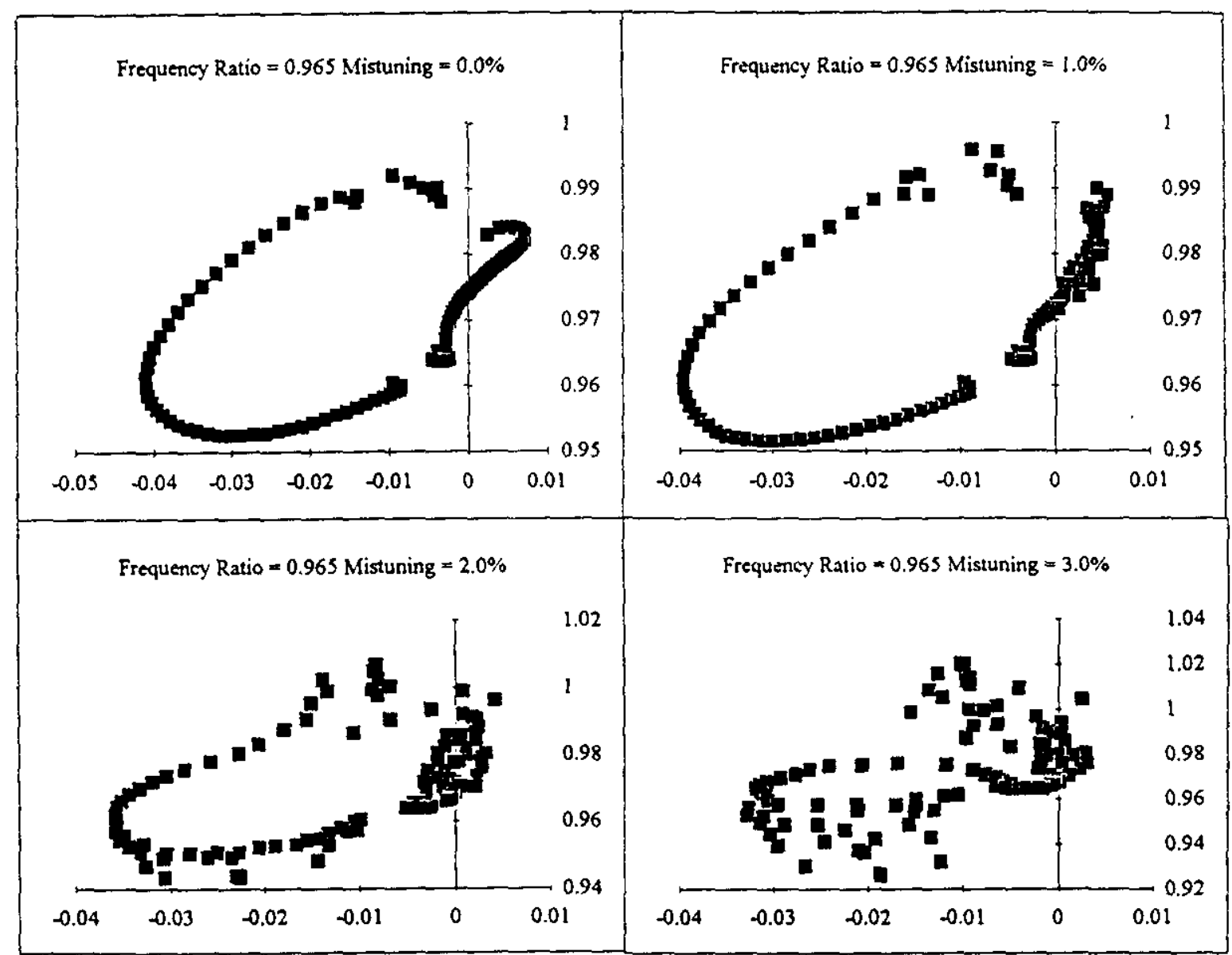

Figure 6. Scattering of the regular structure of the root locus by the introduction of random mistuning in the blade-alone torsion natural frequencies (axes are as in Fig. 1). The blade-alone bending to torsion frequency ratio is 0.965 . Observe that although the mistuning is restricted to the torsion frequencles, the entire locus becomes scattered. 


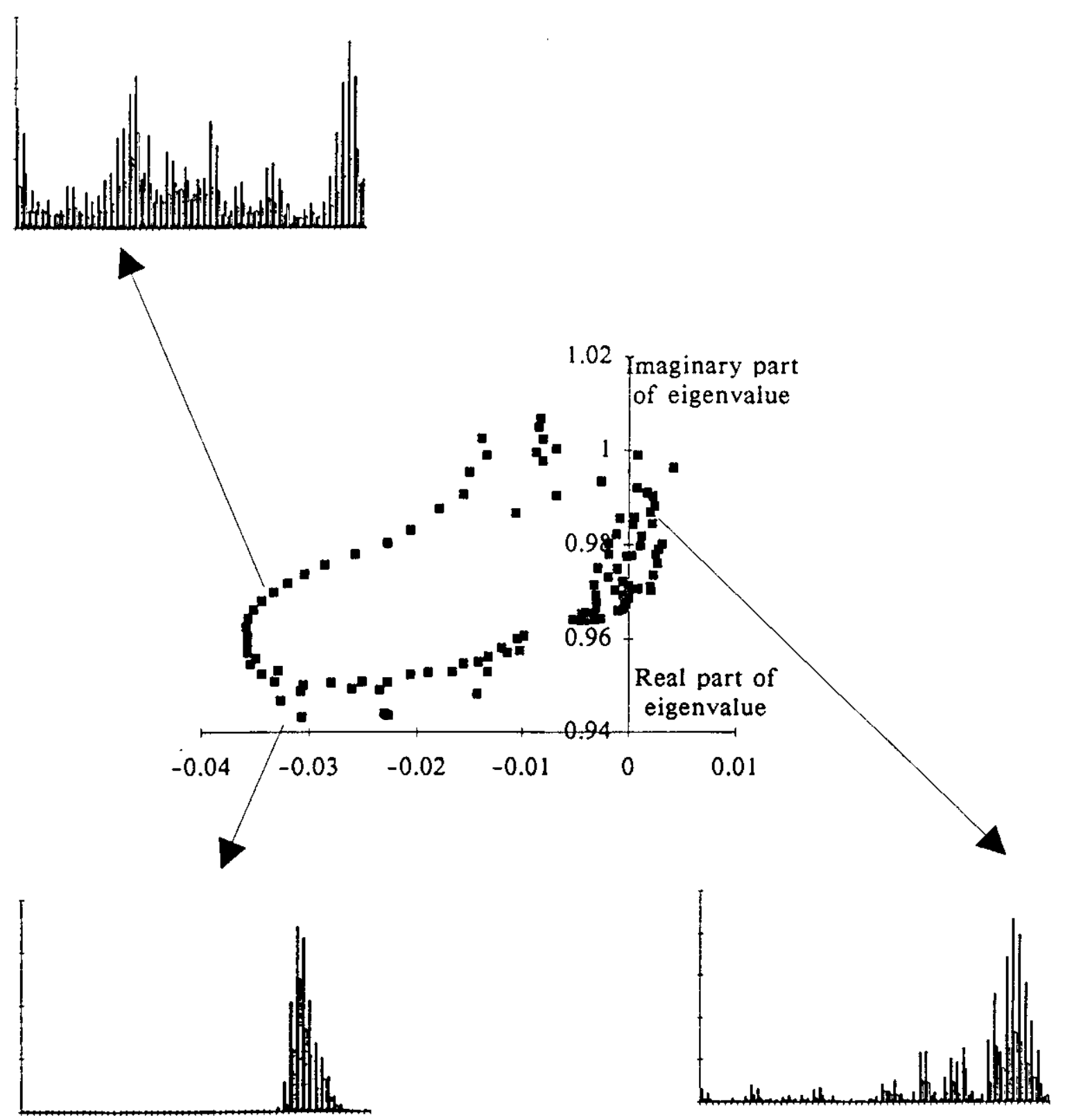

Figure 7. Localization of the mode shapes associated with the scattered root locus. The blade-alone bending to torsion frequency ratio is 0.965 and the random mistuning in the blade-alone torsion natural frequencies has standard deviation 2\%. For the mode shapes, bending amplitudes (black bars) and torsion (white bars) are plotted versus the blade number. 